\title{
Fabrication of depletion type micro-ring modulator with high extinction ratio and high coupling quality factor
}

\author{
Yun Shen ${ }^{1}$, Xiaodong Wang ${ }^{1}$, Wei Zhang ${ }^{1}$, Ciyuan Qiu ${ }^{2}$, Xiulan Cheng ${ }^{1, *}$ \\ ${ }^{1}$ Center for Advanced Electronic Material and Devices, Shanghai Jiao Tong University, Shanghai 200240, China \\ ${ }^{2}$ State Key Lab of Advanced Optical Communication Systems and Networks, Department of Electronic Engineering, Shanghai Jiao Tong \\ University, Shanghai 200240, China
}

\begin{abstract}
We experimentally demonstrate an optical micro-ring modulator based on a reverse-biased PN junction which is integrated into a silicon-on-insulator (SOI) rib waveguide. The performance of micro-ring resonator with different sizes is simulated with Lumerical MODE Solutions. The fabricated device provides a high extinction ratio (ER) of $20 \mathrm{~dB}$ and coupling quality factor (Q) of $2 * 10^{4}$. Modulation rate is higher than $11 \mathrm{Gbit} / \mathrm{s}$ with only $2 \mathrm{~V}$ peak-to-peak (Vpp) driving voltage. The theoretical modulation rate can be higher than $25 \mathrm{Gbit} / \mathrm{s}$ by the optimizing the matching resistor and the test system.
\end{abstract}

\section{Introduction}

High speed electro-optic modulator is a key component in photonics systems for datacom applications. Various mechanisms for realizing highspeed modulation in silicon material have been investigated throughout the years. The main method is altering the refractive index of Silicon, which is based on thermo-optic effect or plasma dispersion effect [4]. The thermo-optic effect is too slow to realize modulation frequencies higher than $1-\mathrm{MHz}$ [8]. Thus, the plasma dispersion effect is considered as the most effective way of refraction index modulation in silicon [4]. Few structures of electro-optic modulator have been reported, such as P-I-N diode in injection type [1-3, 5-6], PN diode in depletion type [7-12, 15-17, 21], MOS capacitor [13] and so on. The mechanism of carrier-injectionbased P-I-N modulator is to change the refraction index of silicon material with the free-carrier dispersion. Its modulation rate is limited due to the slower diffusion rate of minority carriers [2]. However, the carrierdepletion silicon ring modulators employing reversebiased PN diode have been demonstrated to have desirable outstanding performances including high speed higher than $10 \mathrm{~Gb} / \mathrm{s}$, high extinction ratio (ER) more than $5 \mathrm{~dB}$, low peak-to-peak driving voltage $\left(\mathrm{V}_{\mathrm{pp}}\right)$ less than $2 \mathrm{~V}$, and insensitive to carrier lifetime [9], etc.

Most of silicon-based modulators are incorporated into Mach-Zehnder Interferometer (MZI) structures in order to obtain a wider optical bandwidth, high thermal stability, lower chirp, more reasonable tolerance to fabrication variations and no photon cavity lifetime limitation [12]. Compared to MZI structure, the microring resonator structure has some obvious advantages like small footprint, flexible structure, low power consumption, simple fabrication and easier integration.
The modulation rates of depletion type micro-ring modulators have been demonstrated up to $40 \mathrm{Gbit} / \mathrm{s}$ [15, 16]. However, most of the domestic researches on this field are no experiment demonstration due to the limited fabrication condition. In this work, relying on the advanced processing condition of Center for Advanced Electronic Material and Devices (AEMD) in Shanghai Jiao Tong University, we designed and fabricated a depletion type micro-ring modulator integrated with high-index-contrast SOI rib waveguides. The ring resonator exhibits a high ER more than $20 \mathrm{~dB}$ and a high quality factor $(\mathrm{Q})$ more than $2 * 10^{4}$. Modulation rate is higher than $11 \mathrm{Gbit} / \mathrm{s}$ with $2 \mathrm{~V}_{\mathrm{pp}}$ from $-1.6 \mathrm{~V}$ to $0.4 \mathrm{~V}$.

\section{Device modelling}

\subsection{Structure design}

The schematic of the electro-optic modulator is shown Figure 1. The main components in our device include a ring resonator coupled to a single rib waveguide. The input light is injected from a tunable laser into a surface TE mode supported grating coupler which was etched into the waveguide. At the other end of the device, the output light is collected by another grating coupler. The transmission of the waveguide is highly sensitive to the signal wavelength and is greatly reduced at wavelengths in which the ring circumference corresponds to an integer number of guided wavelengths [1]. The resonance wavelength is modified by tuning the effective refractive index of the ring waveguide, inducing a strong modulation of transmitted signal. The effective refractive index of ring is altered by applying different reverse-bias voltages to $\mathrm{PN}$ diode, which changes the width of depletion region of $\mathrm{PN}$ junction.

\footnotetext{
"Corresponding author: : xlcheng@sjtu.edu.cn
} 


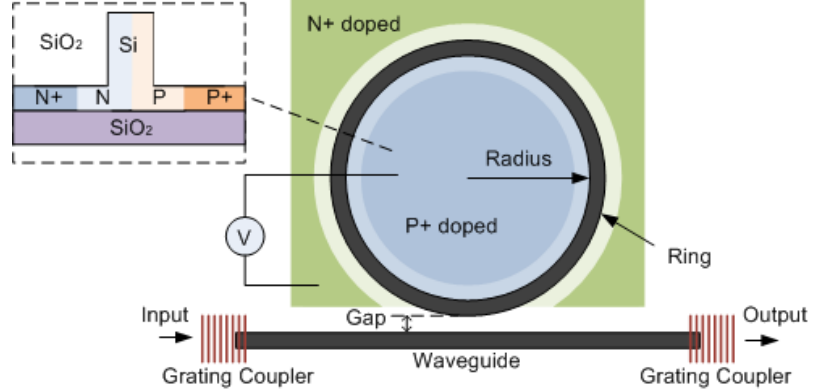

Fig. 1. Schematic layout of the micro-ring modulator.

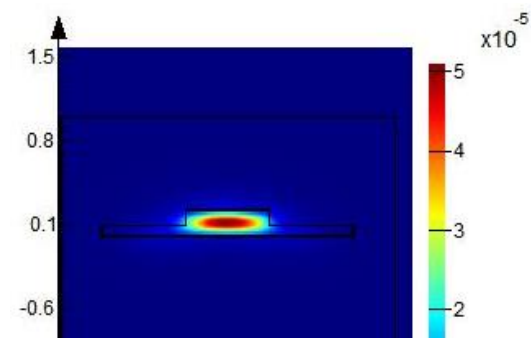

Fig. 2. Simulation about light field distribution(TE0 mode) of rib waveguide.

As shown in Figure 1, Ring resonator has two key parameters, ring radius as well as the gap between ring and straight waveguide. To obtain the best performance of ring resonator, we have simulated several ring resonators with different parameters by Lumerical Mode Solutions. It turned out that ring resonator with $10 \mu \mathrm{m}$ radius and $100 \mathrm{~nm}$ gap performed kind of best around $1.55 \mu \mathrm{m}$ wavelength. However, in actual process, the layout should cover different parameters to ensure that we can get the best one among all the final devices.

The waveguide structure can affect the overall performance of the device. The device uses a rib waveguide with $220 \mathrm{~nm}$ height, $500 \mathrm{~nm}$ width and $90 \mathrm{~nm}$ slab thickness based on SOI substrate, in which only quasi-TE mode was supported [19]. Figure 2 is the simulation about light distribution of ridge waveguide, most of which is TE0 mode (quasi-TE mode). A rib structure leads to vertical confinement of the optical mode as a result of the high refractive index difference between the air/silicon/ $/ \mathrm{SiO}_{2}$ and horizontal confinement results from the etched rib [18].

\subsection{Process simulation}

To achieve strong efficiency modulation, the target ion concentration of $\mathrm{PN}$ junction was $1 \mathrm{E} 18 \mathrm{~cm}^{-3}$. The $\mathrm{P}$ region was doped twice to provide a uniform doping, $5 \mathrm{E} 12 \mathrm{~cm}^{-2}$ of boron with doping energy of $16 \mathrm{KeV}$ and $40 \mathrm{KeV}$ respectively. Similarly, the $\mathrm{N}$ region was doped twice (3E12 $\mathrm{cm}^{-2}$ of phosphorus with doping energy of $45 \mathrm{KeV}$ and $110 \mathrm{KeV}$ respectively). The ohmic contacts were formed by highly doped $\mathrm{P}+\mathrm{N}+$ regions $\left(4 \mathrm{E} 15 \mathrm{~cm}^{-2}\right)$ of boron and phosphorus, suited in the slab on both sides of the waveguide, $0.8 \mu \mathrm{m}$ away from the center of the waveguide. SILVACO has been employed to optimize the overlap between the injected free carriers [18]. Figure 3 is the concentration distribution of ridge waveguide cross section. The color depth indicates the level of ion concentration. It shows that the ion concentration of $\mathrm{P}+$ and $\mathrm{N}+$ region have reached the order of magnitude of $1 \mathrm{E} 20 \mathrm{~cm}^{-3}$.

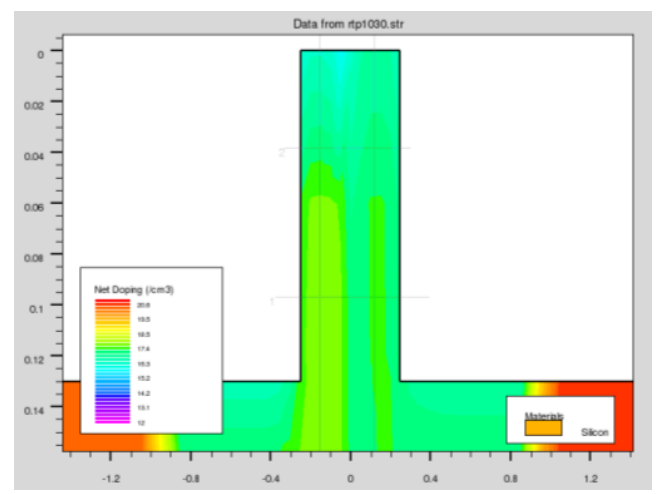

Fig. 3. Concentration distribution in ridge waveguide.

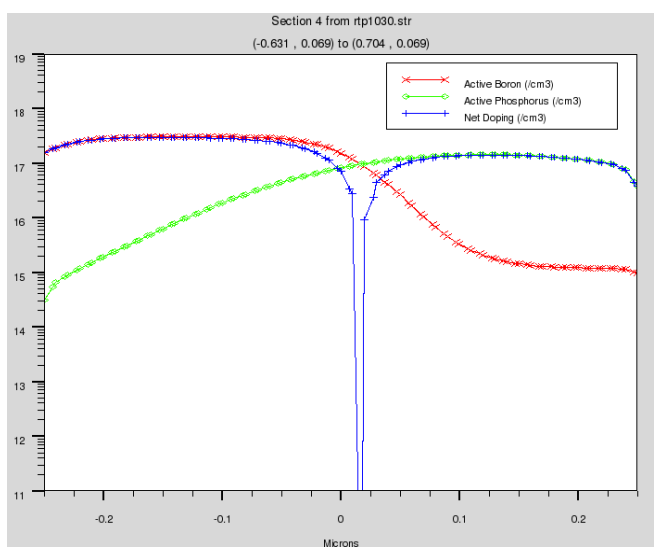

Fig. 4. Concentration curve in waveguide cross section.

Figure 4 shows the concentration curve in waveguide cross section. We can clearly see the depletion region in the middle of waveguide and the concentration distribution is uniform. The depletion region coincides with the main area of the optical mode field, which meets our design requirement.

\section{Device fabrication}

The fabrication process of electro-optical modulator is demonstrated in Figure 5. The process started with a SOI wafer, containing a $3 \mu \mathrm{m}$ thick buried oxide layer and a $220 \mathrm{~nm}$ thick top silicon layer. Firstly, define the alignment markings by deep-UV lithography process, which was then etched into the silicon layer by Inductively Coupled Plasma (ICP) [20]. Except for the alignment marking process, all the other resist windows were defined by Electron-beam lithography system (EBL). Then define the grating coupler by EBL and etch $70 \mathrm{~nm}$ thickness top silicon. Next, etch grooves along the outside of the device to define the straight waveguide and micro-ring resonator region. The grooves are $3 \mu \mathrm{m}$ width and $220 \mathrm{~nm}$ depth (top silicon all etched). Following the etching step, define the slab region by EBL and etch $150 \mathrm{~nm}$ thickness top silicon. To this step, the rib waveguide has been fabricated, as shown in Figure 5(b). 
Then define the $\mathrm{P}+\mathrm{PNN}+$ doping region by four times of EBL and ion implantation with different implantation doses and energies. Firstly, define the resist window of $\mathrm{N}+$ doping region outside the ring and implant phosphorus $\left(4 \mathrm{E} 15 \mathrm{~cm}^{-2}, 30 \mathrm{KeV}\right)$ into the $\mathrm{N}+$ slab region. The $\mathrm{P}+$ doping region (boron, $4 \mathrm{E} 15 \mathrm{~cm}^{2}, 4$ $\mathrm{KeV}$ ) was formed in the same way. To minimise interaction with the optical mode and absorption losses, the gap between the waveguide and the heavy doping region was set to be $0.8 \mu \mathrm{m}$. Then in the same way the other two light doping regions were doped symmetrically to form the PN junction. During doping process, the thickness of photoresist should be at least 2 $\mu \mathrm{m}$. Following the implantation process, rapid thermal annealing (RTP) at $1030{ }^{\circ} \mathrm{C}$ for 8 seconds is needed to activate the doping regions, repair lattice damage and form a uniform P/N type background doping. RTP is better than other annealing processes since it results in a high level of electrical activation whilst minimising dopant diffusion [2]. As illustrated in Figure 5(c), the main structure of ring resonator was completed.

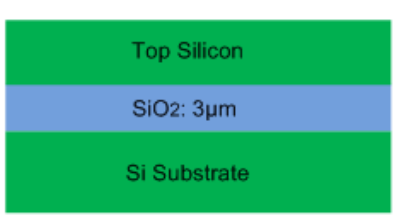

(a) SOI wafer

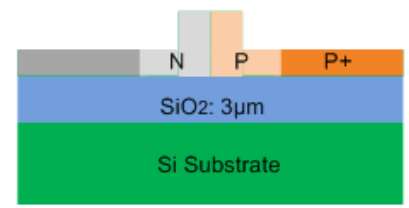

(c) Ion Implantation and RTP annealing

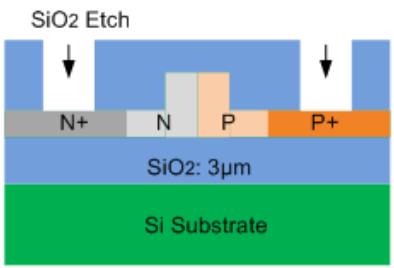

(e) Etch $\mathrm{SiO} 2$

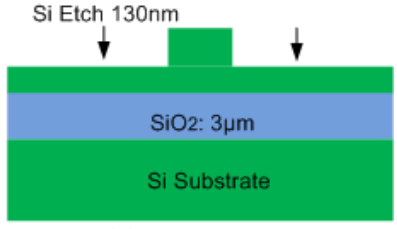

(b) Rib waveguide

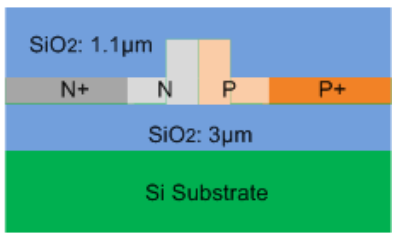

(d) Deposit SiO2 layer

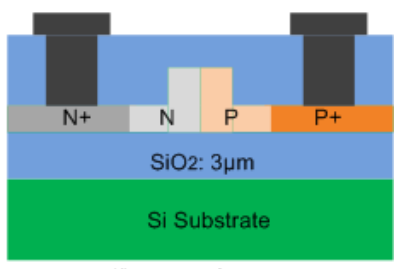

(f) Mental Contact

Fig. 5. Fabrication process of modulator.

The final process is to fabricate the contact electrodes. Its position is critical to the performance of modulator since the frequency response is limited by the RC cut off frequency resulting from capacitive effects within the junction and the resistance of the doped regions and metal contacts [21]. Then the sample was covered by a $1.1 \mu \mathrm{m}$ thick $\mathrm{SiO}_{2}$ layer using plasma-enhanced chemical vapor deposition (PECVD), see Figure 5(d). As shown in Figure 5(e), contact windows were opened in the $\mathrm{SiO}_{2}$ layer above the $\mathrm{P}+$ and $\mathrm{N}+$ doping region by dry etching for $1.0 \mu \mathrm{m}$ and wet etching (HF solution) for $0.1 \mu \mathrm{m}$. Wet etching has advantages such as slower etching rate, better selectivity, which can effectively avoid over etching. The electrodes were formed by lift-off process. The photoresist we used was PMMA (A9), which was spin-coated with a slow rotate speed to provide a thick photoresist layer, enabling the lift-off process easier. After electrode areas defined by EBL, dip it in HF solution for a few seconds to remove the native oxide layer, then a $40 \mathrm{~nm}$ thick titanium layer and a $100 \mathrm{~nm}$ thick TiN film were sputtered by Multi-target Magnetic Control Sputtering System. In the same way $600 \mathrm{~nm}$ thickness of aluminum was sputtered on the TiN film. Dip the sample in acetone solution to remove the photoresist along with the metal layers above. Because the photoresist on electrode areas was developed, the electrodes were not removed while dipping. Finally, these multilayer metallizations were annealed in nitrogen at $450{ }^{\circ} \mathrm{C}$ for 30 minutes, enabling the thin Ti layer to transfer to $\mathrm{TiSi}_{2}$ at the $\mathrm{Si} / \mathrm{Ti}$ interface, resulting in a lower electric resistance. As for the TiN film, it is used as a diffusion barrier in case of interdiffusion of silicon and aluminum during annealing [22]. Figure 5(f) shows the fabricated device cross section.

\section{Experimental results}

A top-view microscope image of the fabricated device is shown in Figure 6. It turned out that a micro-ring resonator with $10 \mu \mathrm{m}$ ring radius and $300 \mathrm{~nm}$ gap space performed best among all the devices. The tunable laser injected light with wavelength from 1530 to $1570 \mathrm{~nm}$ into the waveguide. As demonstrated in Figure 7, this resonator exhibits a free spectra range (FSR) of $9.44 \mathrm{~nm}$, $3 \mathrm{~dB}$ bandwidth of $0.074 \mathrm{~nm}$, ER higher than $20 \mathrm{~dB}$ and high Q better than $2 * 10^{4}$.

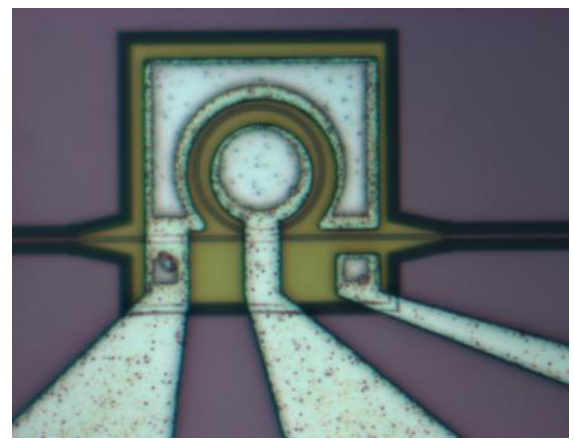

Fig. 6. Microscope top-view image of the fabricated modulator.

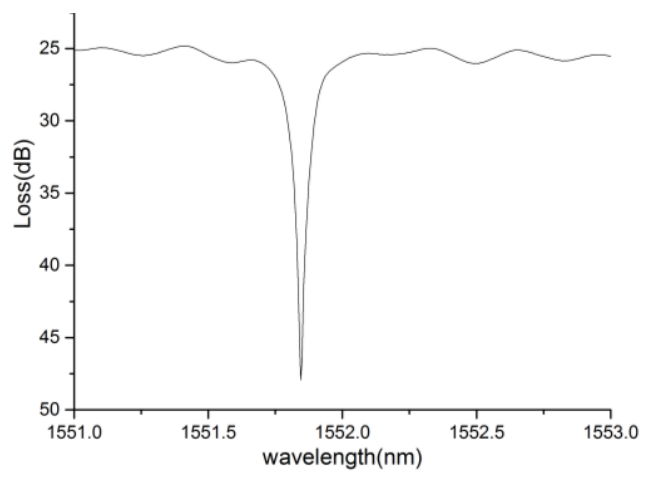

Fig. 7. Transmission spectra of the ring resonator.

The first step was to test the static characteristics of the modulator. Figure 8 shows the measured resonance spectra shift at different DC bias voltages, from $0.7 \mathrm{~V}$ to 
$-3 \mathrm{~V}$. With different reverse-bias voltages applied to the $\mathrm{PN}$ diode, the effective index of rib waveguide was changed due to the drift motion of majority carriers, which made the resonance wavelength shift. Assuming voltage swing from $0 \mathrm{~V}$ to $-1 \mathrm{~V}\left(1 \mathrm{~V}_{\mathrm{pp}}\right)$, we can see in Figure 8 that the resonance shift is about $10 \mathrm{pm}$. The ring modulator demonstrates extinction ratio of $19.5 \mathrm{~dB}$ and quality factor of 11820 at $-2 \mathrm{~V}$ reverse bias voltage. The operation wavelength can be set at around $1549.435 \mathrm{~nm}$.

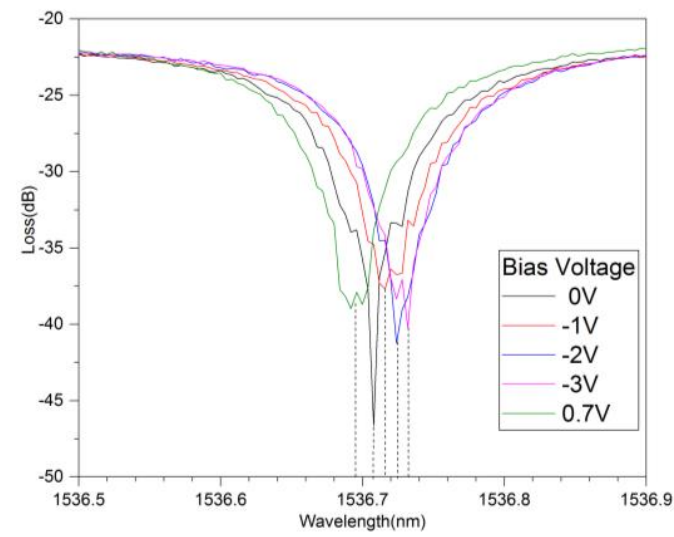

Fig. 8. Transmission spectra with different voltages applied to the PN diode.

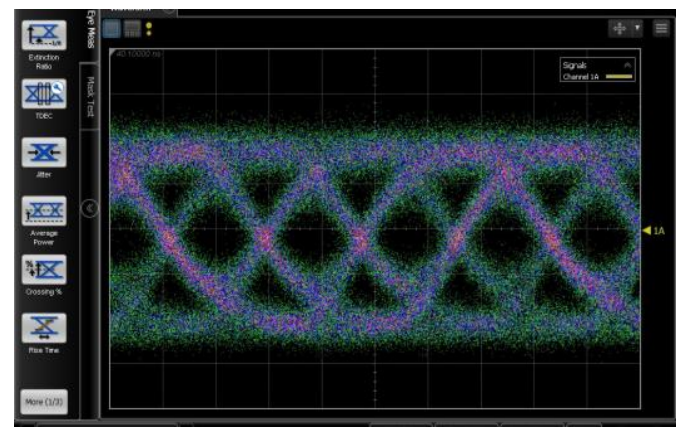

Fig. 9. Eye diagrams obtained by the modulator using 11 $\mathrm{GHz}$ OOK signal with $2 \mathrm{~V}_{\mathrm{pp}}$.

Then we tested the dynamic characteristics of the modulator. $2 \mathrm{~V}_{\mathrm{pp}}$ externally driven voltage (from $-1.6 \mathrm{~V}$ to $0.4 \mathrm{~V}$ ) was applied to the electrodes via the highspeed prober from signal generator. The light was modulated and transferred into electrical signal by photodiode. The electrical signal had the same frequency as driven voltage and its waveform was displayed on the oscilloscope. In the meantime, the eye diagram was obtained. As shown in Figure 9, an open eye can be observed at $11 \mathrm{Gbit} / \mathrm{s}$ with $2 \mathrm{~V}_{\mathrm{pp}}$ swinging from $-1.6 \mathrm{~V}$ to $0.4 \mathrm{~V}$. From the eye diagram, the width of eyeline is slightly wide. It may be caused by the noises since the Erbium Doped Fiber Amplifier (EDFA) was not added into the test system.

\section{Conclusions}

In this work a micro-ring modulator based on the depletion of a PN junction integrated into a SOI rib waveguide at $1.55 \mu \mathrm{m}$ operation wavelength was demonstrated. The detailed fabrication process was illustrated and key procedures were simulated in SIVALCO TCAD. The device exhibits an extinction ratio of more than $15 \mathrm{~dB}$, quality factor more than $10^{4}$ and modulation rate is no less than $11 \mathrm{Gbit} / \mathrm{s}$. Limited by the condition of test system, the modulation rate has not reached its limitation. This is the first attempt to fabricate the silicon micro-ring electro-optic modulator based on a domestic platform. By the optimization of matching resistor, device structure and process parameters, the modulation rate can be higher than 25 Gbit/s.

\section{References}

1. Xu Q. F., Schmidt B, Pradhan S, et al. Nature, 435(2005): 325-327

2. Xu Q. F., Manipatruni S., Schmidt B., et al. Opt. Express, 15(2007): 430-436

3. Manipatruni S, Xu Q. F., Schmidt B., et al. Opt. Express, (2007): 537-538

4. Soref R., Bennett B. Quantum Electron, 23(1987): 123-129

5. F. Gan, F. X. Kartner. Conference on Lasers and ElectroOptics, (2005).

6. L. Zhou, A. W. Poon. Opt. Express, 14 (2006) :6851-6857.

7. A. Liu, L. Liao, D. Rubin, H. Nguyen, B. Ciftcioglu, Y. Chetrit, et al. Opt. Express, 15(2007) : 660-668.

8. F. Y. Gardes, G. T. Reed, N.G. Emerson. Opt. Express, 13(2005) : 8845-8854.

9. Guoliang Li, Xuezhe Zheng, Jin Y, Hiren Thacker, et al. Opt. Express, 19(2011):20435-20443.

10. D. J. Thomson, F. Y. Gardes, et al. Opt. Express, 20(2012) : 12014-12020.

11. D. J. Thomson, F. Y. Gardes, et al. Opt. Express, 19(2011) : 11804-11814.

12. D. J. Thomson, H. Porte, B. Goll, et al. Laser \& Photonics, 8(2014) : 180-187.

13. Liu A S, et a1. Nature, 427(2004) : 615-618.

14. G. T.Reed, G. Z. Machanovich, F Y. Gardes, et al. Nanophotonics, 3(2014).

15. Guoliang Li, Xuezhe Zheng, et al . 2012 IEEE.

16. D. Marris-Morini et al. Opt. Express. 21(2013) :22471-22475.

17. M. Pantouvaki, P. Verheyen, J. De Coster, G. Lepage, et al. Optical Communication, (2015).

18. P. D. Hewitt, G. T. Reed. Lightwave Technology, 18(2000): $443-450$.

19. Qiu C. Y., Ye X., Soref R., Yang L., et al. Opt. Letter, 37(2012) : 3942-3944.

20. Qiu C. Y., Gao W. L., R. Vajtai, et al. NanoLett, 14(2014) : 6811-6815.

21. F.Y. Gardes1, A. Brimont, P. Sanchis2, G. Rasigade, et al. Opt. Express, 17(2009):21986-21991. 